\title{
Screening for Suicide Ideation among Older Primary Care Patients
}

\author{
Marnin J. Heisel, PhD, Paul R. Duberstein, PhD, Jeffrey M. Lyness, MD, and \\ Mitchell D. Feldman, MD, MPhil
}

Objectives: Older adults have high rates of suicide and typically seek care in primary medical practices. Older adults often do not directly or spontaneously report thoughts of suicide, which can impede suicide prevention efforts. Therefore, the use of additional approaches to suicide risk detection is needed, including the use of screening tools. The objective of this study was to assess whether brief screens for depression have acceptable operating characteristics in identifying suicide ideation among older primary care patients and to examine potential sex differences in the screen's accuracy.

Methods: We administered the 15-item Geriatric Depression Scale (GDS), which includes a 5-item GDS subscale (GDS-SI) designed to screen for suicide ideation, to a cross-sectional cohort of 626 primary care patients ( 235 men, 391 women) 65 years of age or older in the Northeastern United States. We assessed presence of suicide ideation with items from the Hamilton Rating Scale for Depression and the Structured Clinical Interview for the Diagnostic and Statistical Manual of Mental Disorders, Fourth Edition.

Results: Patients expressing suicide ideation $(n=69)$ scored higher on the GDS and GDS-SI than those who did not $(n=557)$. A GDS cut score of 4 maximized sensitivity $(0.754)$ and specificity $(0.815)$, producing an area under the curve of $0.844(P<.001)$ and positive and negative predictive values of 0.335 and 0.964 , respectively. Optimal cut scores were 5 for men and 3 for women. A GDS-SI cut score of 1 was optimal for the total sample and for both men and women.

Conclusions: The GDS and GDS-SI accurately identify older patients with suicide ideation. Research is needed to examine their acceptability and barriers to routine use in primary care. $(\mathrm{J}$ Am Board Fam Med 2010;23:260-269.)

Keywords: Suicide Ideation, Death Ideation, Geriatrics, Depression Screening

Later life is a period of heightened risk for suicide; older adults have among the highest rates of suicide of all age groups in the United States. ${ }^{1}$ The 2006 suicide rate for men over the age of 65 years $(28.56$ per 100,000) far exceeds that of the general population $(11.15$ per 100,000$) .^{2}$ Suicide ideation and

This article was externally peer reviewed.

Submitted 1 August 2008; revised 23 July 2009; accepted 28 July 2009.

From the Departments of Psychiatry and Epidemiology \& Biostatistics, Schulich School of Medicine and Dentistry, The University of Western Ontario and Lawson Health Research Institute, London, Canada (MJH); Geriatric Psychiatry Program, Department of Psychiatry, University of Rochester Medical Center, Rochester, New York (PRD, JML); and the Division of General Internal Medicine, University of California, San Francisco (MDF).

Funding: This study was funded in part by United States Public Health Service grant R01 MH061429 (JML); K24 MH071509 (JML); K24 MH072712 (PRD); an American Foundation for Suicide Prevention Young Investigator Award (MJH); and a Canadian Institutes of Health Research New Investigator Award (MJH). desire for death are important targets for risk identification and intervention in the primary care setting $^{3-5}$ because they confer risk for mortality by suicide ${ }^{6,7}$ and other causes. ${ }^{8,9}$ Few older adults atrisk for suicide are seen in mental health settings; most seek treatment in primary care settings ${ }^{10}$ and may even be more likely to do so than nonsuicidal older adults. ${ }^{11}$ Primary care providers are thus well

Prior Presentation: This study was presented at the Annual Conference of the Canadian Association for Suicide Prevention, Toronto, Canada, October 2006.

Conflict of interest: Dr. Lyness participated in a symposium supported by Ingelheim Boehringer but did not personally receive support. Dr. Heisel received an honorarium for helping to develop a clinical treatment guideline and associated knowledge translation tools for the assessment of suicide risk in older adults with the Canadian Coalition for Seniors' Mental Health.

Corresponding author: Marnin J. Heisel, PhD, Department of Psychiatry, The University of Western Ontario, London Health Sciences Centre, 375 South Street, Room \#5054, London, Ontario, N6A 4G5, Canada (E-mail: Marnin. Heisel@lhsc.on.ca). 
positioned to identify high-risk patients and initiate interventions to mitigate suicide-related morbidity and mortality. ${ }^{12}$ Recent multicenter research trials support the effectiveness of collaborative care interventions for reducing and/or resolving suicide ideation among older primary care patients ${ }^{3,13}$; however, barriers exist to the accurate detection of suicide risk and intervention with at-risk older adults.

One study indicated that nearly $40 \%$ of patients 65 years or older who died by suicide had directly reported a wish to die to a medical provider during the prior year. ${ }^{7}$ However, few at-risk older adults, particularly men, ${ }^{14}$ spontaneously report symptoms of distress and/or thoughts of suicide, ${ }^{15}$ which can impede the accurate detection of suicide risk. Primary care physicians often have difficulty asking their patients about sensitive topics such as suicide $^{16-20}$; however, they are more likely to do so with patients who present with symptoms of major depressive disorder or those who request an antidepressant. ${ }^{17}$ Older primary care patients who do not spontaneously report depressive symptoms may not receive needed treatment, necessitating innovative approaches for detecting symptoms of depression, including suicide ideation. No consensus exists as yet on how to identify patients at risk for suicide.

The US Preventive Services Task Force investigated whether screening for suicide risk in primary care settings reduces suicide-related morbidity and mortality, and they discovered a paucity of research addressing this question. ${ }^{21}$ Well-designed studies are needed to explore the accuracy of screening tests for identifying at-risk primary care patients. For screening tests to be viable in primary care settings they need to be brief, valid, easy to administer and score, and written in a fashion that helps initiate sensitive clinical dialogue regarding a patient's desire for death and for suicide. Such an approach may enhance the identification of at-risk suicidal older adults in primary care, increasing the likelihood of initiating life-saving interventions.

Few studies exist that assess the psychometric properties of screens for late-life suicide risk in primary care samples. The available literature about suicide risk detection in primary care tends to report on the use of depression scales rather than dedicated suicide risk assessment instruments. The cost-effectiveness of suicide risk assessment scales as a first-line screening strategy is limited by the relatively low prevalence of suicide ideation among primary care patients. ${ }^{22,23}$ The use of screens that can detect depressed patients and those who may be at risk for suicide serves a dual purpose, potentially reducing costs of measures and time allotted for screening. Empirical findings suggest that primary care practitioners do not typically ask their patients about suicide. ${ }^{17,20}$ The use of more subtle approaches to detecting late-life suicide risk may help overcome clinicians' barriers to initiating difficult patient discussions regarding thoughts of suicide.

Vannoy et $\mathrm{al}^{24}$ reported on the prevalence and course of suicide ideation among participants in the Improving Mood-Promoting Access to Collaborative Treatment trial, a multisite, collaborative care intervention trial for late-life depression. Presence of suicide ideation (14\%) was screened using a single item on the Hopkins Symptoms Checklist instructing patients to indicate: "In the past month, how much were you distressed by thoughts of ending your life?" Options ranged from "a little bit" to "extremely." The authors indicated that assessment of suicide ideation increased the likelihood of receiving effective care; however, they did not report on the operating characteristics of this screening item, such as its sensitivity, specificity, and positive or negative predictive values. This item does not assess the presence of suicide ideation, only of distressing suicide ideation, yet research does not indicate a higher risk for suicide among patients reporting distressing suicide ideation versus any suicide ideation. Hence, use of this item could potentially limit the detection of suicide ideation among those who do not experience or endorse the symptom as distressing.

The 9-item Patient Health Questionnaire (PHQ-9) ${ }^{25}$ is increasingly being used as a screen for depression in primary care. Benefits of this scale include its relative brevity, standardized administration and scoring, and item content reflecting the 9 Diagnostic and Statistical Manual of Mental Disorders-Fourth Edition symptoms of major depressive disorder. ${ }^{26}$ Nonetheless, the measure's suicide ideation item, which instructs patients to report on the frequency of "thoughts that you would be better off dead, or of hurting yourself in some way" during the previous 2 weeks, conflates death ideation with suicide ideation. Little research exists assessing the operating characteristics of the PHQ-9 suicide ideation item with older primary care patients. Sirey and colleagues ${ }^{27}$ reported that 
$13 \%$ of older, home meal-delivery recipients interviewed endorsed suicide ideation on the PHQ-9. They noted an increase in prevalence of suicide ideation with increasing depressive symptom severity and an association with the presence of chronic pain, but noted that some older adults endorsed suicide ideation in the absence of depression. They did not report on the operating characteristics of the suicide ideation item with respect to detecting suicide ideation assessed with an external criterion measure. Donnelly and $\mathrm{Kim}^{28}$ reported on the prevalence of suicide ideation as reported by older Korean adults recruited from a senior's center who completed a Korean translation of the PHQ-9; they focused on positive responses to the options of having suicide ideation for "several days" (8.9\%), "more than half the days" (3.4\%), and "nearly every day" (3.4\%). However, these authors did not report on the operating characteristics of this item with respect to the external criterion of suicide ideation, and so sensitivity, specificity, and positive and negative predictive values were not available for detection of late-life suicide risk using the PHQ-9 suicide ideation item.

In the present study, we assessed the operating characteristics of the Geriatric Depression Scale (GDS), ${ }^{29}$ a common screen for late-life depression, ${ }^{30}$ with reference to presence of suicide ideation in a primary care sample of older adults. Unlike most other depression assessment tools, such as the Hamilton Rating Scale for Depression $(\mathrm{Ham}-\mathrm{D})^{31}$ and the PHQ-9, ${ }^{25}$ the GDS does not assess somatic symptoms of depression. Given that somatic symptoms are common among older adults, the use of depression measures to assess somatic symptoms might artificially inflate the prevalence of late-life depression. ${ }^{29}$ The GDS yes/no response key is easy to score and the measure is available in longer (30-item) and shorter (15-item and 5-item) formats. The GDS does not include an item directly assessing presence of suicide ideation, which is an obvious limitation. However, research indicates that GDS total scores and a 5 -item subscale can effectively differentiate older adults with higher versus lower levels of suicide ideation. ${ }^{32}$ The 5 items of this empirically derived subscale assess perceived hopelessness, worthlessness, emptiness, an absence of happiness, and lack of perception that it is "wonderful to be alive," all of which are variables theoretically and empirically associated with suicide ideation. ${ }^{1,32-34}$ Further- more, research supports more subtle approaches that can be used to assess the presence of suicide ideation among older adults ${ }^{33}$ given their general tendency to downplay the presence and severity of depressive symptoms. ${ }^{14,15}$ Hence, in the present study we assessed whether the GDS ${ }^{29}$ has acceptable operating characteristics in identifying older primary care patients who express a desire for death or for suicide. We also examined potential sex differences in the scale's accuracy given sex differences in older adults' suicide ideation, ${ }^{4,34}$ rates of suicide, ${ }^{2,35}$ and symptom reporting. ${ }^{14,35}$

\section{Methods \\ Sample}

We attempted to recruit all patients 65 years of age or older who presented for care on selected days in the waiting rooms of private practices and university-affiliated clinics in general internal medicine, family medicine, and geriatrics in the Northeastern United States. Interviewers described the study to patients and obtained written, informed consent using procedures approved by a university-based research ethics board. Study safety procedures dictated that when a research interview elicited or raised questions about any type or severity of suicide ideation the rater immediately reviewed the case with an experienced supervising psychiatrist. The psychiatrist then intervened to the extent necessary and allowable per standard clinical practice; the intervention typically included discussion of the concerns with the patient's primary care provider. In principle, patients who were judged to be at imminent risk of self-harm could have been brought in for emergency psychiatric evaluation at an appropriate local facility, but this proved not to be necessary for any patient during this study.

\section{Measures and Procedures}

The severity of patients' depressive symptoms was initially assessed at the time of study recruitment with the 15 -item version of the self-report GDS. ${ }^{29}$ Blind to patient GDS scores, interviewers later administered the Structured Clinical Interview for the Diagnostic and Statistical Manual of Mental Disorders, Fourth Edition (SCID) ${ }^{36}$ and the 24item, examiner-rated Ham-D. ${ }^{31}$ Patients also completed a demographic measure and trained raters conducted brief assessments of cognitive functioning, ${ }^{37}$ physical functioning, ${ }^{38,39}$ and medical bur- 
Table 1. Geriatric Depression Scale-Suicide Ideation Screening Items*

\begin{tabular}{ll}
\hline GDS Item No. & \multicolumn{1}{c}{ Item } \\
\hline 3 & Do you feel that your life is empty? \\
7 & Do you feel happy most of the time? \\
11 & $\begin{array}{l}\text { Do you think it is wonderful to be alive? } \\
12\end{array}$ \\
14 & $\begin{array}{l}\text { Do you feel pretty worthless the way you } \\
\text { are now? }\end{array}$ \\
& Do you feel that your situation is \\
\hline
\end{tabular}

*Scoring for the Geriatric Depression Scale (GDS) items involves assigning a response of "yes" or "no" to each item. These items were drawn from the GDS. ${ }^{29}$

den ${ }^{40}$; the latter was completed by a physicianinvestigator (JML) based on information from the patient interview and review of the primary care medical records. In the present study, we examined patient scores on the GDS and the GDS-SI (see Table 1), an internally consistent $(\alpha=0.68)$, 5 -item suicide ideation screen derived from the $\mathrm{GDS}^{32}$ comprising items assessing perceived hopelessness (\#14); worthlessness (\#12); emptiness (\#3); happiness (\#7, reverse-scored); and perception that it is "wonderful to be alive" (\#11, reversescored). We previously derived the GDS-SI items by investigating individual GDS items that were significantly correlated with presence and intensity of suicide ideation, which were assessed using the Geriatric Suicide Ideation Scale and the Scale for Suicide Ideation as reported by a heterogeneous sample of adults 65 years of age and older who were recruited from medical, mental health, residential, and community sites. ${ }^{32}$ The 5 items comprising the GDS-SI were each significantly associated with suicide ideation in that sample and demonstrated strong internal consistency (Cronbach's $\alpha, 0.82$ ). We have adopted this same scale to assess its cut score for presence of suicide ideation in an older primary care patient sample.

In the present study we have used the term suicide ideation to refer to a desire for death and/or for suicide; we operationalized this construct as a score of $\geq 1$ on the Ham-D item assessing thoughts of death or of suicide and/or $\geq 2$ on a similar item in the SCID Mood Disorder Module. We categorized patients as suicide ideators based on endorsement of either the relevant Ham-D or SCID item (Ham-D $\geq 1$ or SCID $\geq 2$ ). We chose to create a composite measure of suicide ideation to increase the likelihood of identifying patients with suicide ideation given limitations associated with singleitem measurement and recognizing that these measures have overlapping, although distinct, time frames; the Ham-D assesses depressive symptoms during the previous week and the SCID assesses mood disorder symptoms during the previous month. We selected a relatively low level of severity of suicide ideation for our analyses to enhance detection of those patients desiring death and/or suicide. We recognized that older adults with suicide ideation who are reluctant to express thoughts of suicide to a provider might be more willing to endorse a somewhat less intense form of the symptom. ${ }^{33}$ Those not endorsing either item were classified as nonideators.

\section{Statistical Analysis}

Demographic data were summarized using counts, means, standard deviations, and percentages. Differences between groups on continuous measures were computed using independent-sample $t$ tests; equal variances were not assumed when the Levene test for the equality of variances was statistically significant. Internal consistency reliability was assessed using Cronbach alpha coefficient $(\alpha)$. Operating characteristics of the GDS were evaluated by computing sensitivity and specificity statistics for cut scores identified using receiver operating characteristic (ROC) curve analysis; positive predictive values and negative predictive values were also computed. We tested statistical significance of the ROC curves by computing the area under the curve (AUC); to do this we plotted sensitivity against 1-specificity and compared those areas against a null hypothesis of $50 \%$ coverage. Consistent with this study's purpose of assessing the feasibility of screening for suicide ideation in an older primary care population and with its broad goal of encouraging primary care clinicians to discuss thoughts of death, dying, and suicide with their older patients, we compared patients with suicide ideation versus all patients rather than restricting our analyses to those with depression. All reported $P$ values are 2-tailed, with $\alpha$ set at 0.05 unless otherwise indicated. Analyses were computed using SPSS software version 16.0 for Windows (SPSS, Inc., Chicago, IL).

\section{Results}

Of 1415 individuals approached in medical practice waiting rooms for study enrollment, 704 (49.8\%) 
Table 2. Demographic and Clinical Characteristics of Study Participants $(n=626)$

\begin{tabular}{|c|c|c|c|}
\hline Variable & $\mathrm{n}(\%)$ & Mean (Standard Deviation) & Range \\
\hline Age (in years) & & $75.02(6.78)$ & $65-95$ \\
\hline \multicolumn{4}{|l|}{ Sex } \\
\hline Men & $235(38)$ & & \\
\hline Women & $391(62)$ & & \\
\hline \multicolumn{4}{|l|}{ Marital Status } \\
\hline Married & $326(53)$ & & \\
\hline Separated/Divorced & $65(11)$ & & \\
\hline Widowed & $193(31)$ & & \\
\hline Single/Never Married & $31(5)$ & & \\
\hline Missing Data & 11 & & \\
\hline \multicolumn{4}{|l|}{ Race } \\
\hline White & $584(93)$ & & \\
\hline Black & $29(5)$ & & \\
\hline Other & $11(2)$ & & \\
\hline Don't Know & $2(<1)$ & & \\
\hline Lives Alone & $217(36)$ & & \\
\hline Years of Education & & $14(2.63)$ & \\
\hline \multicolumn{4}{|l|}{ Employment Status } \\
\hline Retired & $516(82)$ & & \\
\hline Unemployed/Disability Benefits & $10(2)$ & & \\
\hline Part- or Full-Time Employment & $90(14)$ & & \\
\hline Part- or Full-Time Student & $3(<1)$ & & \\
\hline Missing Data & 7 & & \\
\hline CIRS & & $7.61(3.90)$ & $1-68$ \\
\hline IADL & & $1.96(3.81)$ & $0-22$ \\
\hline PSMS & & $1.61(2.32)$ & $0-20$ \\
\hline Number of prescription medications & & $7.46(3.67)$ & $0-24$ \\
\hline \multicolumn{4}{|l|}{ Presence of medical condition } \\
\hline Arthritis & $400(64)$ & & \\
\hline Cancer & $68(11)$ & & \\
\hline Neurologic disorder & $132(21)$ & & \\
\hline COPD & $86(14)$ & & \\
\hline Hypothyroidism & $133(21)$ & & \\
\hline Decreased hearing & $168(27)$ & & \\
\hline Decreased vision & $46(7)$ & & \\
\hline Diabetes & $117(19)$ & & \\
\hline Hypertension & $453(72)$ & & \\
\hline Cardiovascular disease & $181(29)$ & & \\
\hline
\end{tabular}

CIRS, Cumulative Illness Rating Scale total scores; IADL, Instrumental Activities of Daily Living Scale; PSMS, Physical SelfMaintenance Scale; COPD, chronic obstructive pulmonary disease.

completed intake assessments. Enrollees did not differ from those who did not enroll in age, sex, or GDS score, based on available data. ${ }^{41,42}$ For these analyses we excluded patients who scored $\leq 15$ on the Mini Mental State Examination (MMSE; $\mathrm{n}=$ $4)^{37}$ and those who had incomplete data $(n=74 ; 51$ were missing GDS data, 18 were missing the Ham-D suicide ideation item, 3 were missing SCID suicide ideation data, and 2 were missing
MMSE data); this yielded 626 patients in the final sample. Patient demographic characteristics appear in Table 2.

Two hundred twenty-one patients met Diagnostic and Statistical Manual of Mental Disorders, Fourth Edition, criteria for an active mood disorder (35\%), including 109 for major depressive disorder (17\%) and 108 for minor depression (17\%). Patients had mean scores of 2.4 (SD, 2.74; range, 
$0-13$ ) on the GDS; mean scores of 8.8 (SD, 6.33; range, $0-37)$ on the Ham-D; and mean scores of 27.7 (SD, 2.30; range, 17-30) on the MMSE. Overall, 69 patients $(11 \%)$ reported suicide ideation on the combined suicide ideation variable and 557 (89\%) did not; 41 (6.5\%) endorsed the Ham-D suicide ideation item and 62 (9.9\%) endorsed the SCID suicide ideation item. Concordance was strong between the 2 suicide ideation items (591 of 626 patients; $94.4 \%$ ). No significant sex differences emerged for the prevalence of suicide ideation; women endorsed a nonsignificantly higher prevalence of the symptom than did men overall $(\mathrm{n}=49$ $[12.5 \%]$ vs $\left.\mathrm{n}=20[8.5 \%] ; \chi^{2}, 2.42 ; P=.12\right)$ as well as on the Ham-D $(\mathrm{n}=30$ [7.7\%] vs $\mathrm{n}=11$ [4.7\%]; $\left.\chi^{2}, 2.15 ; P=.14\right)$ and SCID suicide ideation items $\left(\mathrm{n}=43[11 \%]\right.$ vs $\left.\mathrm{n}=19[8.1 \%] ; \chi^{2}, 1.40 ; P=.24\right)$. When compared with those patients with no suicide ideation, patients with suicide ideation scored significantly higher on the GDS (mean, 6.19 and $\mathrm{SD}, 3.57$ vs mean, 1.92 and $\mathrm{SD}, 2.21 ; \mathrm{t}_{(75)}, 9.71$; $P<.001$ ); on the Ham-D (mean, 16.52 and SD, 6.74 vs mean, 7.68 and $\mathrm{SD}, 5.28 ; \mathrm{t}_{(79)}, 10.51 ; P<$ .001 ; excluding the suicide ideation item); and on the 5-item GDS-SI (mean, 1.96 and SD, 1.54 vs mean, 0.29 and SD, 0.68; $\left.\mathrm{t}_{(71)}, 8.88 ; P<.001\right)$. These data reflect the strong association between late-life depression and suicide. ${ }^{1}$

ROC curve analyses next evaluated the sensitivity and specificity of GDS and GDS-SI cut scores for suicide ideator versus nonideator group status, in the total sample, and by patient sex (see Table 3). All ROC curves were significant and each had an AUC exceeding $80 \%$. A GDS cut score of 4 maximized sensitivity (0.754) and specificity (0.815) for the total sample; cut scores of 5 and 3 were optimal for men and for women. A GDS-SI cut score of 1 maximized sensitivity and specificity for group status for the total sample $(0.797$ and 0.804 , respectively), for men (0.800 and 0.809), and for women (0.796 and 0.801).

\section{Discussion}

Older adults at risk for suicide often present to primary care medical providers for care days to weeks before suicide ${ }^{10,11}$; however, barriers exist to the detection of a patient's suicide risk, including a paucity of research about effective screens for latelife suicide risk and provider discomfort when addressing the complex and sensitive topic of suicide with older patients. ${ }^{16-20}$ The present findings highlight the potential promise of screening for late-life suicide ideation with brief, validated depression scales in primary care practices and thus provide one approach for broaching this difficult topic with at-risk older patients. The 15-item GDS and 5-item GDS-SI accurately differentiated older primary care patients who expressed suicide ideation from those who did not, suggesting that this screen may effectively identify individuals for whom a more in-depth suicide risk assessment would be warranted. The GDS-SI demonstrated sensitivity and specificity equivalent to that of the 15 -item GDS in terms of identifying people at risk for suicide. Despite sex differences in suicide rates, ${ }^{2,35}$ study patients did not report a significant difference in the prevalence of suicide ideation and results yielded a consistent GDS-SI cut score for men and for women; this suggests a complex association between suicide ideation and ultimate death by suicide. Busy clinicians wishing to quickly screen for possible suicide risk may thus prefer using this brief tool.

Study findings were limited to older primary care patients and to the specific measures used. Cut scores are influenced by population prevalence; investigation of late-life suicide ideation in alternate treatment settings could have yielded different cut scores. ${ }^{43}$ We deliberately selected a relatively low threshold for suicide risk as a minimum presence of the desire for death because we recognized that frequent thoughts of death among older primary care patients can signal presence of health-related concerns, end of life issues, depression, and suicide risk. A higher threshold might have produced different cut scores. Positive predictive values for detecting suicide ideation, although relatively low, were consistent with those reported in the literature about screening for late-life suicide ideation. $^{44,45}$ The study group was predominantly white and relatively well-educated for an older cohort; findings may not generalize to other groups. The internal consistency of the GDS-SI was acceptable and may have been limited somewhat by the small number of items in this subscale. This was a secondary analysis of data from a study exploring the naturalistic course of late-life depression and was not initially designed to assess cut scores for suicide risk; thus, data about patient lifetime suicide attempt history were unavailable for analysis. The relatively high prevalence of major depressive dis- 


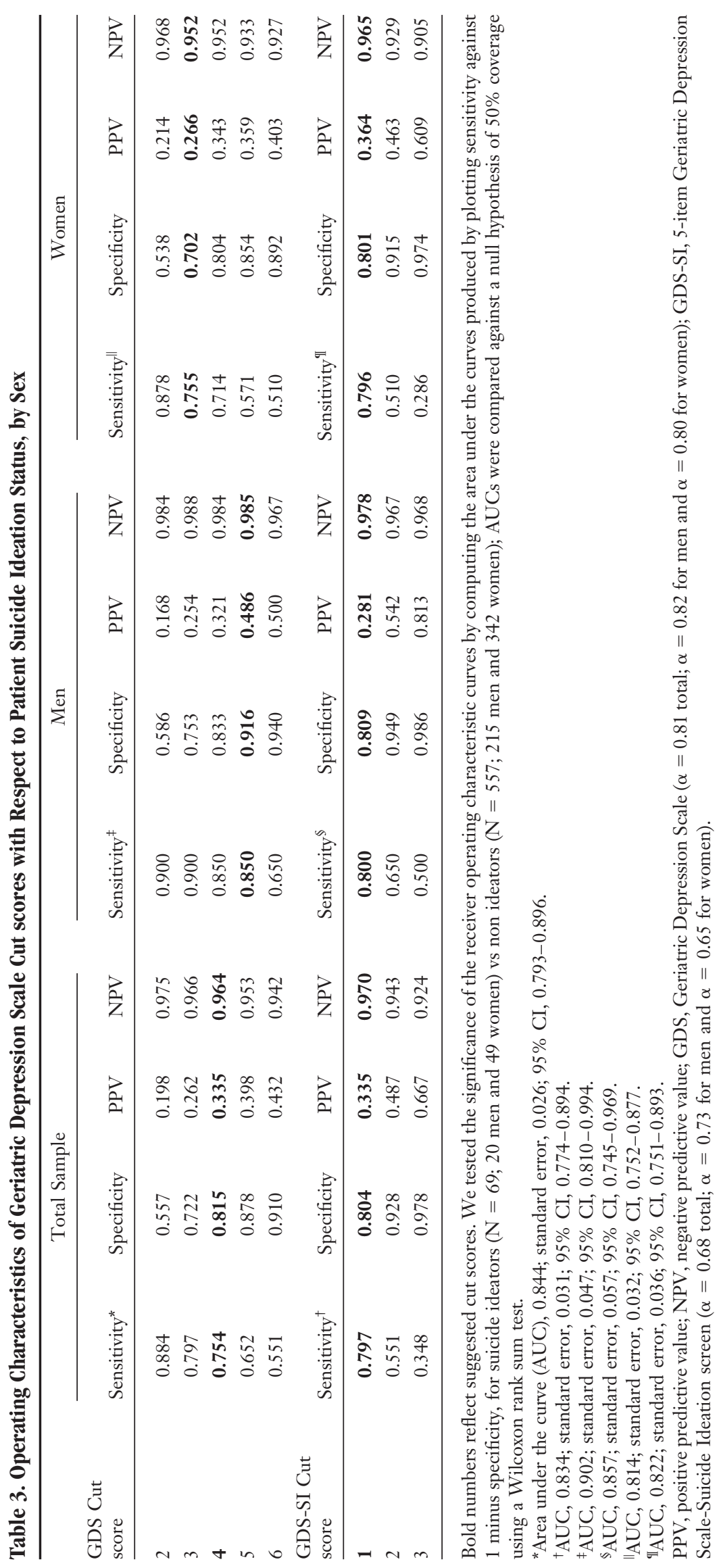


order in this study's sample reflects our inclusion of partially remitted episodes of depression and is thus consistent with prior work in primary care settings, particularly work that uses an "inclusive" approach to ideational and somatic symptoms of depression as recommended by a multi-institutional working group in relation to research on late-life depression. ${ }^{46}$ Findings may differ in samples with different rates of major depressive disorder. Trained interviewers collected data in the context of a research study.

Further studies are needed to compare the utility of the GDS with other instruments such as the PHQ-9. ${ }^{25}$ Research is needed to assess the validity of brief screens with respect to longer multidimensional measures of presence and severity of suicide ideation than the Ham-D and SCID suicide ideation items, such as the Geriatric Suicide Ideation Scale. ${ }^{33}$ Research is also needed to compare tools that more subtly screen for suicide ideation, such as the GDS-SI, with items that more directly assess the presence and intensity of suicide ideation. Outcomes research is needed to examine the prediction of suicidal behavior among older adults who screen positive for suicide ideation over the long term.

Empirical findings suggest that primary care practitioners do not typically ask their patients about suicide. ${ }^{17,20}$ Researchers have questioned the utility of incorporating routine screening for depression into primary health care but have noted an increased effectiveness of depression screening when it is incorporated into collaborative care programs for the treatment of depression. ${ }^{47}$ Providers may be reluctant to engage older patients in discussions about suicide risk if they lack knowledge of available crisis and mental health resources and/or familiarity with empirical evidence that supports collaborative models of primary and mental health care in reducing late-life suicide risk. Therefore, we suggest an approach that is more subtle and designed to help clinicians overcome barriers to initiating difficult discussions regarding patient thoughts of suicide. Collaborative care has proven effective in enhancing the detection of depression, increasing the uptake of mental health services, resolving depressive episodes, decreasing rates of suicide ideation, and reducing mortality in older adults. ${ }^{3,13,48,49}$ Research is needed to investigate the feasibility and acceptability of incorporating routine screening for suicide ideation in primary care by clinicians and clinic staff and whether the use of validated scales enhances clinician comfort in discussing these sensitive issues with older adults and improves detection of those at risk for suicide.

It is important for clinicians to feel comfortable following up positive screens with a more in-depth assessment of a patient's suicide risk. Suggestions for doing so in clinical practice involve beginning the discussion by asking less threatening questions, such as questions about the patient's current circumstances, social network, activities of daily living, sleep, appetite, and other physical health symptoms, then gradually moving into questions assessing mental health difficulties, including questions about mood symptoms, misuse of alcohol and prescription medications, hopelessness, tiredness of life, the wish to die, suicide ideation, and the presence of a suicide plan. ${ }^{16,50}$ Although not specific to older adults, research supports the use of screening measures in detecting and responding to suicide risk in patients receiving treatment for depression. ${ }^{51}$ Patients at risk for suicide require ongoing clinical attention and support. Providers are strongly encouraged to help at-risk older adults develop safety plans, listing personal and professional supports to whom they can reach out when they feel desperate, hopeless, and/or at risk for suicide, including telephone distress lines and crisis and emergency services. Clinicians are advised to familiarize themselves with sources of crisis and mental health care support in their communities and with available treatment guidelines. These strategies have not yet been empirically investigated among older adults; however, they are consistent with recent practice guidelines for the assessment of suicide risk and prevention of suicide among older adults. ${ }^{52}$ Screening for suicide ideation in primary care settings may be controversial, ${ }^{53}$ but the need for innovative techniques to enhance dialogue about patient desire for death and suicide in primary care settings is incontrovertible. To facilitate difficult discussions about thoughts of death or suicide, family physicians might consider administering a brief questionnaire containing items that have been carefully selected to identify older patients at risk for suicide. Older patients scoring $\geq 4$ on the GDS (or $\geq 5$ for men or $\geq 3$ for women) and/or $\geq 1$ on the GDS-SI may be excellent candidates for these discussions. Although the GDS and GDS-SI can both be used to identify older patients with suicide ideation, the GDS-SI may be preferable because it is briefer and less 
susceptible to sex differences in symptom reporting. Patients who screen positive may require more in-depth mental health assessments to determine the need for referral and treatment.

We thank the patients, staff, and providers of the following primary care practices: University of Rochester Medical Center, Departments of Medicine and Family Medicine; Pulsifer Medical; East Ridge Family Medicine; RGH Twig Center; Olsan Medical; Clinton Crossings Medical; Wilson-Lifetime; Panorama Internal Medicine; HH Geriatrics; and Culver Medical. K. Gibson, C. Bowen, J. Evinger, A. Niculescu, J. Sauvain, J. Scheltz, and J. Woodhams assisted in data collection.

\section{References}

1. Heisel MJ, Duberstein PR. Suicide prevention in older adults. Clinical Psychology: Science and Practice 2005;12:242-59.

2. Centers for Disease Control and Prevention, $\mathrm{Na}-$ tional Center for Injury Prevention and Control. Web-based Injury Statistics Query and Reporting System fatal injury reports. Available from http:// webappa.cdc.gov/sasweb/ncipc/mortrate.html. Accessed 6 January 2010.

3. Bruce ML, Ten Have TR, Reynolds CF 3rd, et al. Reducing suicidal ideation and depressive symptoms in depressed older primary care patients: a randomized controlled trial. JAMA 2004;291:1081-91.

4. Bartels SJ, Coakley E, Oxman TE, et al. Suicidal and death ideation in older primary care patients with depression, anxiety, and at-risk alcohol use. Am J Geriatr Psychiatry 2002;10:417-27.

5. Pfaff JJ, Almeida OP. Detecting suicidal ideation in older patients: identifying risk factors within the general practice setting. Br J Gen Pract 2005;55: 269-73.

6. Miller M. Geriatric suicide: the Arizona study. Gerontologist 1978;18(5 Pt 1):488-95.

7. Waern M, Beskow J, Runeson B, Skoog I. Suicidal feelings in the last year of life in elderly people who commit suicide. Lancet 1999;354:917-8.

8. Dewey ME, Davidson IA, Copeland JR. Expressed wish to die and mortality in older people: a community replication. Age Ageing 1993;22:109-13.

9. Maier H, Smith J. Psychological predictors of mortality in old age. J Gerontol B Psychol Sci Soc Sci 1999;54:P44-54.

10. Luoma JB, Martin CE, Pearson JL. Contact with mental health and primary care providers before suicide: a review of the evidence. Am J Psychiatry 2002;159:909-16.

11. Juurlink DN, Herrmann N, Szalai JP, Kopp A, Redelmeier DA. Medical illness and the risk of suicide in the elderly. Arch Intern Med 2004;164:1179-84.

12. Rutz W. Preventing suicide and premature death by education and treatment. J Affect Disord 2001;62: $123-9$.
13. Unützer J, Tang L, Oishi S, et al. Reducing suicidal ideation in depressed older primary care patients. J Am Geriatr Soc 2006;54:1550-6.

14. Allen-Burge R, Storandt M, Kinscherf DA, Rubin EH. Sex differences in the sensitivity of two selfreport depression scales in older depressed inpatients. Psychol Aging 1994;9:443-5.

15. Duberstein PR, Conwell Y, Seidlitz L, Lyness JM, Cox C, Caine ED. Age and suicidal ideation in older depressed inpatients. Am J Geriatr Psychiatry 1999; 7:289-96.

16. Cooper-Patrick L, Crum RM, Ford DE. Identifying suicidal ideation in general medical patients. JAMA 1994;272:1757-62.

17. Feldman MD, Franks P, Duberstein PR, Vannoy S, Epstein R, Kravitz RL. Let's not talk about it: suicide inquiry in primary care. Ann Fam Med 2007;5:412-8.

18. Feldman MD, Franks P, Epstein RM, Franz CE, Kravitz RL. Do patient requests for antidepressants enhance or hinder physicians' evaluation of depression? A randomized controlled trial. Med Care 2006; 44:1107-13.

19. Nutting PA, Dickinson LM, Rubenstein LV, Keeley $\mathrm{RD}$, Smith JL, Elliott CE. Improving detection of suicidal ideation among depressed patients in primary care. Ann Fam Med 2005;3:529-36.

20. Kaplan MS, Adamek ME, Rhoades JA. Prevention of elderly suicide: physicians' assessment of firearm availability. Am J Prev Med 1998;15:60-4.

21. Gaynes BN, West SL, Ford CA, Frame P, Klein J, Lohr KN. Screening for suicide risk in adults: a summary of the evidence for the US Preventive Services Task Force. Ann Intern Med 2004;140:822-35.

22. Callahan CM, Hendrie HC, Nienaber NA, Tierney WM. Suicidal ideation among older primary care patients. J Am Geriatr Soc 1996;44:1205-9.

23. Kim YA, Bogner HR, Brown GK, Gallo JJ. Chronic medical conditions and wishes to die among older primary care patients. Int J Psychiatry Med 2006;36: $183-98$.

24. Vannoy SD, Duberstein P, Cukrowicz K, Lin E, Fan $\mathrm{M}-\mathrm{Y}$, Unützer J. The relationship between suicide ideation and late-life depression. Am J Geriatr Psychiatry 2007;15:1024-33.

25. Spitzer RL, Kroenke K, Williams JB. Validation and utility of a self-report version of PRIME-MD: the PHQ primary care study. Primary Care Evaluation of Mental Disorders. Patient Health Questionnaire. JAMA 1999;282:1737-44.

26. American Psychiatric Association. Diagnostic and statistical manual of mental disorders, $4^{\text {th }}$ ed. Washington, DC: American Psychiatric Association; 1994.

27. Sirey JA, Bruce ML, Carpenter M, et al. Depressive symptoms and suicidal ideation among older adults receiving home delivered meals. Int J Geriatr Psychiatry 2008;23:1306-11.

28. Donnelly PL, Kim KS. The Patient Health Questionnaire (PHQ-9K) to screen for depressive disor- 
ders among immigrant Korean American elderly. Journal of Cultural Diversity 2008;15:24-9.

29. Sheikh JL, Yesavage JA. Geriatric Depression Scale: recent evidence and development of a shorter version. In: Clinical gerontology: a guide to assessment and intervention. Brink TL, ed. New York: Howarth Press; 1986:165-73.

30. Stiles PG, McGarrahan JF. The Geriatric Depression Scale: a comprehensive review. J Clin Geropsychol 1998;4:89-110.

31. Williams JB. A structured interview guide for the Hamilton Depression Rating Scale. Arch Gen Psychiatry 1988;45:742-7.

32. Heisel MJ, Flett GL, Duberstein PR, Lyness JM. Does the geriatric depression scale (GDS) distinguish between older adults with high versus low levels of suicidal ideation? Am J Geriatr Psychiatry 2005;13:876-83.

33. Heisel MJ, Flett GL. The development and initial validation of the Geriatric Suicide Ideation Scale. Am J Geriatr Psychiatry 2006;14:742-51.

34. Scocco P, Meneghel G, Caon F, Dello Buono M, De Leo D. Death ideation and its correlates: survey of an over-65-year-old population. J Nerv Ment Dis 2001;189:210-8.

35. Krug EG, Dahlberg LL, Mercy JA, Zwi AB, Lozano R. World Report on Violence and Health. Geneva: World Health Organization; 2002.

36. First MB, Spitzer RL, Gibbon M, Williams JBW. Structured clinical interview for DSM-IV axis I disorders: patient edition. New York: New York State Psychiatric Institute; 1997.

37. Folstein MF, Folstein SE, McHugh PR. "Mini-mental state." A practical method for grading the cognitive state of patients for the clinician. J Psychiatr Res 1975;12:189-98.

38. Lawton MP, Brody EM. Instrumental Activities of Daily Living (IADL) Scale. Original observer-rated version. "Does do" form-for women only. Psychopharmacol Bull 1988;24:785-7.

39. Lawton MP, Brody EM. Physical Self-Maintenance Scale (PSMS): Original observer-related version. Psychopharmacol Bull 1988;24:793-4.

40. Linn BS, Linn MW, Gurel L. Cumulative illness rating scale. J Am Geriatr Soc 1968;16:622-6.

41. Schoepflin Sanders ML, Lyness JM, Eberly S, King DA, Caine ED. Cerebrovascular risk factors, executive dysfunction, and depression in older primary care patients. Am J Geriatr Psychiatry 2006;14:14552.
42. Lyness JM, Kim J, Tang W, et al. The clinical significance of subsyndromal depression in older primary care patients. Am J Geriatr Psychiatry 2007;15: 214-23.

43. Sackett DL, Haynes RB. The architecture of diagnostic research. BMJ 2002;324:539-41.

44. Awata S, Bech P, Koizumi Y, et al. Validity and utility of the Japanese version of the WHO-Five Well-Being Index in the context of detecting suicidal ideation in elderly community residents. Int Psychogeriatr 2007;19:77-88.

45. Fujisawa D, Tanaka E, Sakamoto S, Neichi K, Nakagawa A, Yutaka O. The development of a brief screening instrument for depression and suicidal ideation for elderly: the Depression and Suicide Screen. Psychiatry Clin Neurosci 2005;59:634-8.

46. Lyness JM, Bruce ML, Koenig HG, et al. Depression and medical illness in late life: report of a symposium. J Am Geriatr Soc 1996;44:198-203.

47. Gilbody S, Sheldon T, Wessely S. Should we screen for depression? BMJ 2006;332:1027-30.

48. Bartels SJ, Coakley EH, Zubritsky C, et al. Improving access to geriatric mental health services: a randomized trial comparing treatment engagement with integrated versus enhanced referral care for depression, anxiety, and at-risk alcohol use. Am J Psychiatry 2004;161:1455-62.

49. Gallo JJ, Bogner HR, Morales KH, Post EP, Lin JY, Bruce ML. The effect of a primary care practicebased depression intervention on mortality in older adults. Ann Intern Med 2007;146:689-98.

50. Grek A. Clinical management of suicidality in the elderly: an opportunity for involvement in the lives of older patients. Can J Psychiatry 2007;52(6 Suppl 1):47S-57S.

51. Duffy FF, Chung H, Trivedi M, Rae DS, Regier DA, Katzelnick DJ. Systematic use of patient-rated depression severity monitoring: is it helpful and feasible in clinical psychiatry? Psychiatr Serv 2008;59: $1148-54$

52. Canadian Coalition for Seniors' Mental Health. National guidelines for seniors' mental health: the assessment of suicide risk and prevention of suicide. Toronto: Canadian Coalition for Seniors' Mental Health; 2006.

53. American Psychiatric Association. Practice guideline for the assessment and treatment of patients with suicidal behaviors. Available form http:// www.psychiatryonline.com/pracGuide/pracGuide Topic_14.aspx. Accessed 6 January 2010. 